\title{
Control of drinking-bout magnitude in schedule-induced polydipsia by interpellet-interval duration
}

\author{
WILLIAM J. FREED \\ Laboratory of Clinical Psychopharmacology, \\ National Institute of Mental Health, Washington, D.C. 20032 \\ and \\ JOSEPH MENDELSON \\ University of Kansas, Lawrence, Kansas 66045
}

\begin{abstract}
Schedule-induced polydipsia (SIP) is a function of interpellet-interval (IPI) durations: intermediate (40-180-sec) IPIs are most effective in producing SIP, while longer and shorter IPIs are increasingly less effective. In order to determine whether each IPI exerts its influence on SIP by regulating drinking during that IPI itself or during the immediately succeeding IPI, rats were presented with systematic sequences of IPI durations during individual sessions of SIP testing. It was found that a very brief $(20-\mathrm{sec})$ IPI tended to reduce the duration of drinking during the ongoing IPI, but that a very long (4-min) IPI reduced the probability that any drinking would occur during subsequent IPIs. It was suggested that the motivation to drink is reduced following long IPIs, while feeding, or behaviors anticipatory to feeding, compete with drinking when IPI lengths are very short.
\end{abstract}

Schedule-induced polydipsia (SIP) refers to a pronounced enhancement of drinking by food-deprived animals when they are intermittently given small morsels of food (Falk, 1971). Numerous studies have established that intermediate degrees of feeding intermittency are most effective in producing SIP. Interpellet-interval (IPI) durations in the range of from 40 to $180 \mathrm{sec}$ generally seem to be optimal, while longer and shorter durations are increasingly less effective (Bond, 1973; Falk, 1966; Flory, 1971; Hawkins, Schrot, Githens, \& Everett, 1972; Keehn \& Colotla, 1971).

Why are very long and very short IPI durations less effective? It has been suggested that SIP is reduced when IPI lengths are short simply because feeding competes successfully with drinking under these conditions (Cohen, 1975; Freed, Zec, \& Mendelson, 1977). However, this explanation has not generally been accepted, because even during very short IPIs rats drink during only a fraction of the amount of time that is available for drinking. We hoped that the present approach would help to elucidate this problem.

Given that SIP is characterized by drinking bouts that usually occur following the delivery of each food

W. J. Freed's complete address is: Laboratory of Clinical Psychopharmacology; National Institute of Mental Health, SMR, IRP; William A. White Building, St. Elizabeths Hospital, Washington, D.C. 20032. pellet (Falk, 1971; Flory \& O'Boyle, 1972; Gilbert, 1974), it might be expected that long and short IPIs are ineffective either because drinking is reduced following very long or very short IPIs (i.e., as a function of the duration of the immediately preceding IPI) or because drinking is depressed during very long or very short IPIs (i.e., as a function of the expected delay to the delivery of the next food pellet). A third possibility is that individual IPI durations do not directly determine specific drinkingbout magnitudes, but that SIP is related only to the average duration of all the IPIs of a test session.

To study these possibilities experimentally, we presented rats, in a SIP situation, with systematic sequences of IPI durations during individual testing sessions. We used two different repeating sequences of IPI durations: a brief $(20-\mathrm{sec})$ IPI followed by two 80 -sec IPIs (the 20-80A-80B sequence), and a long $(240-\mathrm{sec})$ IPI followed by two 80 -sec IPIs (the 240-80A-80B sequence). Through this tactic, we hoped to determine whether long $(240-\mathrm{sec})$ and short $(20-\mathrm{sec})$ IPIs would cause decreased drinking during the long and short IPIs themselves or during the 80 -sec IPIs that were to follow these long and short IPIs.

\section{METHODS}

\section{Subjects}

Six adult female Sprague-Dawley rats that had been used in a previous experiment (Freed \& Mendelson, 1977) served as 
subjects. These animals were individually housed on a constant day-night cycle with free access to water. Food was restricted so as to maintain them at $80 \%$ of their free-feeding body weights.

\section{Apparatus}

The animals were tested in two similar Plexiglas boxes that have been described previously (Freed \& Mendelson, 1977). Each contained a water-filled drinking tube and, on the opposite wall, a food cup. Contacts with the drinking tubes were detected by means of Grason-Stadler drinkometers that delivered approximately $.7 \mu \mathrm{A}$ to the animals with each contact.

\section{Procedure}

Noyes food pellets $(45 \mathrm{mg})$ were delivered noncontingently according to fixed-time schedules. Initially, each animal was tested for two 66.7-min sessions with IPI durations alternating between 20 and $80 \mathrm{sec}$. For the next 10 sessions, pellets were delivered so that IPI durations occurred in the 20-80A-80B sequence. This sequence was repeated 20 times per session. In order to acclimate the animals to the $240-\mathrm{sec}$ IPI, they were given two 213.3-min sessions with alternating 240- and 80-sec IPIs. For the next six or seven sessions, the pellets were delivered so that the IPI durations occurred cyclically in the $240-80 \mathrm{~A}-80 \mathrm{~B}$ sequence. This sequence was also repeated 20 times per session.

Chamber illumination (15-W incandescent lamp) was used as a cue to signal IPI duration. For three of the animals, the light was on only during all 20 - and $240-\mathrm{sec}$ IPIs, and for the other three animals, the light was on only during $80-\mathrm{sec}$ IPIs.

\section{Data Collection and Analysis}

Durations of drinking were automatically summed on three separate running-time meters, one for each kind of IPI (e.g., 20 and $240 ; 80 \mathrm{~A}$ or $80 \mathrm{~B}$ ). Percentages of IPIs which contained drinking were determined by inspection of event-marker records. For each session, data were analyzed for each type of IPI in terms of (1) the mean drinking duration per IPI, determined by dividing the duration of drinking by the number of IPIs that contained some drinking, and in terms of (2) the percentage of IPIs during which at least some drinking occurred (pct IPIs). Data were analyzed statistically, for purposes of illustration, by correlated t tests.

\section{RESULTS}

During the 20-80A-80B sequence, each animal exhibited the typical postpellet drinking pattern. The animals ingested a mean of $27 \mathrm{ml}$ of water per session. The behavior of the animals did not tend to change over the course of testing in this condition. There were no differences in pct IPIs for the 20 , $80 \mathrm{~A}$, and $80 \mathrm{~B}$ IPIs (Table 1). In contrast, the mean drinking durations were consistently shorter during the 20 -sec IPIs than for either the $80 \mathrm{~A}$ or $80 \mathrm{~B}$
IPIs [ $\mathrm{t}(5)=5.39$ and 10.01 , both ps $<.004]$. This tendency was evident in each of the animals (Figure 1); there was only one session of one animal (the first session for rat No. 4) in which the mean drinking duration during the 20 -sec IPIs exceeded that for either type of 80-sec IPI. Overall, the mean drinking duration during 20 -sec IPIs was about one-half of that during the 80 -sec IPIs $(50 \%$ for $80 \mathrm{~A}$ and $47 \%$ for $80 \mathrm{~B}$ )

A second effect noted in some animals was that drinking durations were slightly shorter during $80 \mathrm{~A}$ than during 80B IPIs (see Figure 1). This was significant for three animals [No. 3, $\mathrm{t}(9)=5.12$, $\mathrm{p}=.0009$; No. $6, \mathrm{t}(9)=5.25, \mathrm{p}=.0008$; No. 7 , $\mathrm{t}(9)=3.08, \mathrm{p}=.013]$ but not for a fourth [No. 8, $t(9)=1.93, p=.083]$. Of the two other animals tested, one drank for longer durations during the $80 \mathrm{~A}$ intervals $[$ No. $5, t(9)=5.60, \mathrm{p}=.0006]$ and the other showed no consistent trend $[$ No. $4, t(9)=$ $0.91, \mathrm{p}=.390 \mathrm{]}$.

During the $240-80 \mathrm{~A}-80 \mathrm{~B}$ sequence, the primary effects observed involved pct IPIs rather than drinking durations. Drinking durations could only be evaluated reliably for four animals. (Only Animals $3,5,6$, and 7 drank consistently during each type of IPI.) Overall, these durations somewhat exceeded those for the $20-80 \mathrm{~A}-80 \mathrm{~B}$ conditions, but were similar for each type of IPI (Table 1).

The pct IPIs during the $240-80 \mathrm{~A}-80 \mathrm{~B}$ condition tended to be reduced overall as compared to the 20-80A-80B condition; this reduction was most marked during the $80 \mathrm{~A}$ and $80 \mathrm{~B}$ IPIs (Table 1). As Figure 2 shows, for five of the six animals, the pct IPIs was less during the $80 \mathrm{~A}$ than during the $240-\mathrm{sec}$ intervals $[$ No. $4, t(5)=19.76, p<.0001$; No. 5, $\mathrm{t}(6)=2.59, \mathrm{p}=.040 ;$ No. $6, \mathrm{t}(6)=7.92, \mathrm{p}=.0005$; No. $7, \mathrm{t}(5)=3.16, \mathrm{p}=.025$; No. $8, \mathrm{t}(4)=12.83$, $\mathrm{p}=.0009]$. The remaining animal drank during a greater percentage of IPIs for the $80 \mathrm{~A}$ than for the 240-sec IPIs [No. 3, $t(5)=2.88, p=.034$ ]. The pct IPIs was similar for the 80A and 80B IPIs, except that one rat drank during a greater percentage of 80B than 80A IPIs [No. 8, $t(4)=4.71, p=.011$ ].

To rule out the possibility that the differences in pct IPIs were due to the greater opportunity to drink during 240-sec IPIs (as compared to 80-sec IPIs), the data were also examined for 240 -sec IPIs that con-

Table 1

Mean (and Standard Error of the Mean) Drinking Durations and Percentages of Interpellet Intervals (IPIs) During Which Drinking Occurred

Type of IPI

\begin{tabular}{|c|c|c|c|c|c|c|c|c|c|c|c|c|}
\hline & \multicolumn{6}{|c|}{ 20-80A-80B Condition } & \multicolumn{6}{|c|}{ 240-80A-80B Condition } \\
\hline & \multicolumn{2}{|c|}{$20 \mathrm{sec}$} & \multicolumn{2}{|c|}{$80 \mathrm{~A}$} & \multicolumn{2}{|c|}{$80 \mathrm{~B}$} & \multicolumn{2}{|c|}{$240 \mathrm{sec}$} & \multicolumn{2}{|c|}{$80 \mathrm{~A}$} & \multicolumn{2}{|c|}{$80 \mathrm{~B}$} \\
\hline & Mean & SE & Mean & $\mathrm{SE}$ & Mean & $\mathrm{SE}$ & Mean & SE & Mean & $\mathrm{SE}$ & Mean & SE \\
\hline Duration & 9.88 & 1.20 & 19.7 & 1.12 & 20.9 & 1.67 & 26.1 & 1.46 & 27.5 & 3.16 & 24.6 & 2.22 \\
\hline Percent IPIs & 83 & 4.1 & 85 & 6.8 & 81 & 6.8 & 66 & 10 & 49 & 20 & 51 & 16 \\
\hline
\end{tabular}




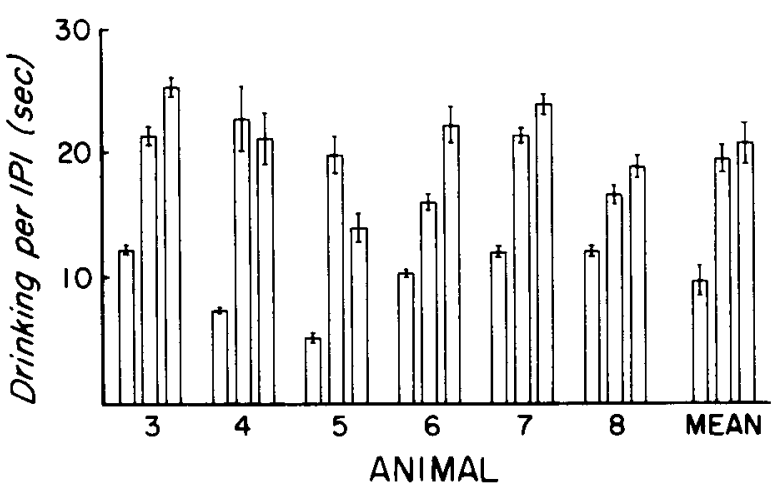

Figure 1. Mean duration of drinking $\left(\overline{\mathbf{X}}_{ \pm}\right.$S.E.M. $)$per interpellet interval for each animal and the mean for all of the animals for the 20-80A-80B condition. These durations were determined by dividing the total duration of drinking during each session by the number of interpellet intervals that contained drinking during that session. The first bar of each group of three represents the 20 -sec intervals, the second bar the $80 \mathrm{~A}$ intervals (80-sec intervals that immediately followed the 20 -sec intervals), and the third bar represents the $80 \mathrm{~B}$ intervals (80-sec intervals that immediately preceded the 20 -sec intervals).

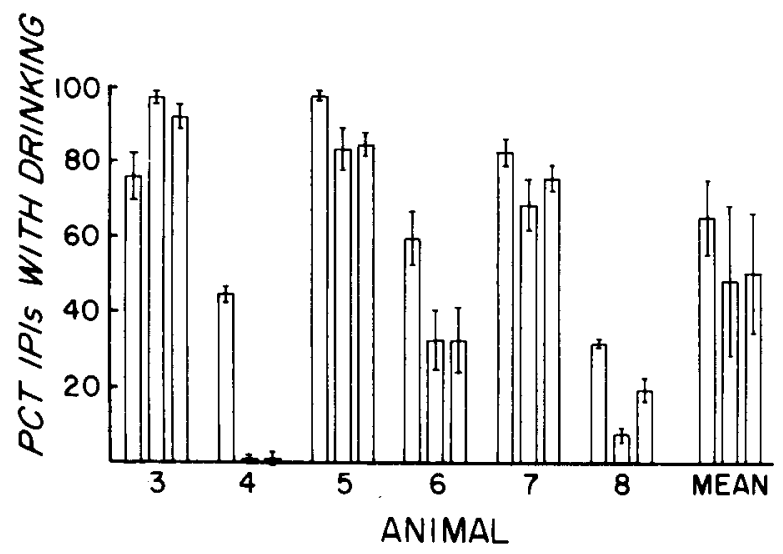

Figure 2. Percentages of IPIs that contained drinking ( $\bar{X}_{ \pm}$ S.E.M.) for each animal, and the mean for all of the animals, for the $240-80 \mathrm{~A}-80 \mathrm{~B}$ condition. The first bar of each triplet represents the 240-sec intervals, the second bar the $80 \mathrm{~A}$ intervals (80-sec intervals that immediately followed the 240 -sec intervals), and the third bar the $80 \mathrm{~B}$ intervals ( $80-\mathrm{sec}$ intervals that immediately preceded the 240 -sec intervals).

tained drinking during the latter part of the 240 -sec intervals but not during the first $80 \mathrm{sec}$. There were only three (of a possible 494) IPIs with this pattern of drinking, one being found for each of Animals 4,5 , and 6. A reanalysis of the data in these terms did not change the statistical findings and did not substantially change the means and standard errors displayed in Figure 2.

\section{DISCUSSION}

During the 20-80A-80B condition, drinking durations were decreased consistently by about $50 \%$ during the short $(20-\mathrm{sec})$ IPIs as compared to the 80 -sec IPIs. There was also a tendency in four of the six rats for drinking durations to be shorter during the $80 \mathrm{~A}$ than during the $80 \mathrm{~B}$ IPIs. No significant differences in pct IPIs were observed. This is consistent with a previous finding that drinking durations are shorter during 15 -sec than during 120-sec IPIs when these IPI durations are alternated (Colotla, Keehn, \& Gardner, 1970).

During the $240-80 \mathrm{~A}-80 \mathrm{~B}$ condition, there were no differences of any substance in drinking durations between the various types of IPIs. However, there was a strong tendency for pct IPIs to be reduced, predominantly during the $80 \mathrm{~A}$ and $80 \mathrm{~B}$ IPIs. It is suggested that $240-\mathrm{sec}$ intervals reduced the tendency to drink during succeeding IPIs. This influence apparently lasted for more than one IPI, as the reductions were still evident (although in one case reduced) during the $80 \mathrm{~B}$ IPIs.

Keehn and Colotla (1971) have hypothesized that the SIP decrement induced by short IPIs is a consequence of decreased durations of drinking bouts, while the SIP decrement induced by long IPIs is due to reductions in the number of drinking bouts, i.e., the frequency with which pellet consumption is followed by drinking. The present results confirm this hypothesis and suggest a mechanism of action. It appears that short IPIs decrease drinking durations during the ongoing IPIs, while long IPIs decrease the probability that any drinking at all will occur during subsequent IPIs. Perhaps feeding or behaviors anticipatory to feeding compete successfully with drinking during short IPIs (Cohen, 1975; Freed et al., 1977). In other words, for short IPIs, while the animal is drinking during each IPI, it perceives that the delivery of the next pellet is imminent. Then the tendency to prepare for consumption of the new pellet, or to eat it, successfully competes with the tendency to continue to drink. Thus there is no need to invoke a motivational deficit to account for the decreased SIP observed during short IPIs; the drinking decrement can be accounted for simply in terms of a competing-response mechanism. On the other hand, in order to explain the drinking deficit observed with long IPIs, it seems necessary to invoke a motivational deficit (Cohen, 1975). Specifically, a long IPI reduces the probability that drinking will occur following consumption of the next food pellet, thus resulting in a reduction in pet IPIs, as was observed in the present experiment. But if on any occasion the post pellet motivation to drink surpasses the drinking threshold so that the animal begins to drink, the usual amount of water will be consumed despite the long IPI, i.e., there will be no reduction in the duration of the drinking bout; this was also observed in the present experiment.

\section{REFERENCES}

Bond, N. Schedule-induced polydipsia as a function of the con summatory rate. Psychological Record, 1973, 23, 377-382. 
CohfN, I. L. The reinforcement value of schedule-induced drinking. Journal of the Experimental Analysis of Behavior, 1975, 23, 37-44.

Colotla, V. A., Keehn, J. D., \& Gardner, L. L. Control of schedule-induced drink durations by interpellet intervals. Psychonomic Science, 1970, 21, 137-139.

Falk, J. L. Schedule-induced polydipsia as a function of fixedinterval length. Journal of the Experimental Analysis of Behavior, 1966, 9, 37-39.

F ALK, J. L. The nature and determinants of adjunctive behavior. Physiology \& Behavior, 1971, 6, 577-588.

FloRY, R. K. The control of schedule-induced polydipsia: Frequency and magnitude of reinforcment. Learning and Motivation, 1971, 2, 215-227.

FloRY, R. K., \& O'Boyle, M. K. The effect of limited water availability on schedule-induced polydipsia. Physiology \& Behavior, 1972, 8, 147-149.

Freed, W. J., \& Mendelson, J. Water intake-volume regulation in the rat: Schedule-induced drinking compared to waterdeprivation-induced drinking. Journal of Comparative and Physiological Psychology, 1977, 91, 569-573.
Freed, W. J., Zec, R. F., \& Mendeison, J. Schedule-induced polydipsia: The role of orolingual factors and a new hypothesis. In J. A. W. M. Weijnen \& J. Mendelson (Eds.), Drinking behavior: Oral stimulation, reinforcement, and preference. New York: Plenum, 1977.

Gilbert, R. M. Ubiquity of schedule-induced polydipsia. Journal of the Experimental Analysis of Behavior, 1974, 21, 277-284.

Hawkins, T. D., Schrot, J. R., Githens, S. H., \& Everett, P. B. Schedule-induced polydipsia: An analysis of water and alcohol ingestion. R. M. Gilbert \& J. D. Keehn (Eds.), Schedule effect: Drugs, drinking, and aggression. Toronto: University of Toronto Press, 1972.

KеенN, J. D., \& Colotla, V. A. Schedule-induced drinking as a function of interpellet interval. Psychonomic Science, 1971, $23,69-71$.

(Received for publication January 9, 1979; revision accepted April 19, 1979.) 